\title{
Effects of anxiety on task switching: Evidence from the mixed antisaccade task
}

\author{
Tahereh L. Ansari, Nazanin Derakshan, And Anne Richards \\ Birkbeck College, University of London, London, England
}

\begin{abstract}
According to the attentional control theory of anxiety (Eysenck, Derakshan, Santos, \& Calvo, 2007), anxiety impairs performance on cognitive tasks that involve the shifting function of working memory. This hypothesis was tested using a mixed antisaccade paradigm, in which participants performed single-task and mixed-task versions of the paradigm. The single task involved the completion of separate blocks of anti- and prosaccade trials, whereas in the mixed task, participants completed anti- and prosaccade trials in a random order within blocks. Analysis of switch costs showed that high-anxious individuals did not exhibit the commonly reported paradoxical improvement in saccade latency, whereas low-anxious individuals did. The findings are discussed within the framework of attentional control theory.
\end{abstract}

There is behavioral evidence to show that anxiety is associated with an attentional bias for threat-related material (see Bar-Haim, Lamy, Pergamin, BakermansKranenburg, \& van IJzendoorn, 2007, for a review). Recent neuroimaging work has also shown that anxiety selectively facilitates early processing of threat and enhances distractibility to task-irrelevant stimuli. According to Bishop (2007; see also Bishop, Duncan, Brett, \& Lawrence, 2004), anxiety is associated with enhanced amygdala activation and reduced recruitment of prefrontal cortical areas (especially the dorsal lateral prefrontal cortex [DLPFC] and the ventral lateral prefrontal cortex [VLPFC]) that are heavily involved in top-down regulation of attention, especially when attentional focus is required for efficient task performance. Both behavioral and neuroimaging work has shown that anxiety is associated with adverse effects on cognitive performance, especially on tasks that require attentional focus. In an attempt to explain the role of attentional control in anxiety and cognitive performance, the attentional control theory of anxiety was put forward by Eysenck, Derakshan, Santos, and Calvo (2007).

The attentional control theory (Eysenck et al., 2007) is a major development of processing efficiency theory (Eysenck \& Calvo, 1992). Based on Baddeley's (1986; see also Derryberry \& Reed, 2002) working memory model, the theory claims that anxiety disrupts the balance between what Corbetta and Shulman (2002) distinguished as the stimulus-driven (involved in bottom-up control, influenced by salient environmental stimuli) and the goaldirected (involved in top-down control, influenced by the current goal) systems. These two systems are generally thought to interact in their functioning (Pashler, Johnston, \& Ruthruff, 2001), but anxiety is believed to increase the influence of the stimulus-driven system over the goaldirected processes, reducing attentional control.

Predictions of the attentional control theory are based on a fundamental distinction by the processing efficiency theory (Eysenck \& Calvo, 1992) between performance effectiveness and performance efficiency. Effectiveness refers to an individual's competence in doing a task (measured by response accuracy), and efficiency refers to the amount of processing resources invested in doing the task (measured by response latency). The theory predicts that anxiety has a greater impact on performance efficiency of tasks requiring the inhibition ("one's ability to deliberately inhibit dominant, automatic, or prepotent responses when necessary"; Miyake et al., 2000, p. 57) and/or the shifting ("shifting back and forth between multiple tasks"; Miyake et al., 2000, p. 55) functions of the central executive. In inhibition, attentional control prevents attentional resources from being allocated to task-irrelevant stimuli, and in shifting, attentional control is used in a positive way to allocate attentional resources to execute the task relevant to the current goal.

A general assumption of the attentional control theory (Eysenck et al., 2007) is that attentional control involves the use of the processing resources of the central executive. However, an alternative proposal is that there are rather specific resource pools and that those involved in attentional control differ from those involved in other processing functions of the central executive (Wickens, 1984). The assumption within attentional control theory is more parsimonious than the one based on a multipleresource approach. However, it is accepted that further research will be required to elucidate the underlying mechanisms involved.

Derakshan, Ansari, Hansard, Shoker, and Eysenck (in press) examined the relationship between anxiety and in- 
hibitory processes, using the antisaccade paradigm (Hallet, 1978). In this task, top-down attentional control is exercised to suppress a reflexive saccade toward an abrupt peripheral stimulus (i.e., inhibit) and to generate a volitional saccade to its mirror position (antisaccade). Performance on this task is compared with that on the prosaccade task, which eliminates the conflict between reflexive and volitional processes by requiring participants to look at the abrupt cue when it appears. As well as making more directional errors, individuals are generally slower to make a correct saccade away from the abrupt stimuli on antisaccade trials, as compared with prosaccade trials (see Hutton \& Ettinger, 2006 , for a review). In the antisaccade task, competition occurs between reflexive saccades (prepotent response) and the antisaccade (volitional response) (Massen, 2004). To predict correct antisaccade performance, Ettinger et al. (2008) identified specific brain regions involved in attentional control. In a neuroimaging study, they found enhanced activation in the supramarginal gyrus, DLPFC, and VLPFC on correct antisaccade trials and interpreted the activation in these areas as indicative of a major involvement in the inhibition of reflexive saccades. Furthermore, it was found that the DLPFC and the VLPFC were also involved in volitional saccade generation.

Derakshan et al. (in press) reported that high-anxious (HA) individuals had longer correct antisaccade latencies than did low-anxious (LA) individuals but that the two groups did not differ on the prosaccade task, suggesting that anxiety is associated with the inhibitory component of attentional control. There was no difference between the two groups in terms of saccadic error rate. These results indicated that anxiety affects efficiency and not effectiveness. Given the involvement of the DLPFC and the VLPFC in correct antisaccade performance, it is reasonable to assume that these areas will be most affected by anxiety and its modulation in attentional control, as assessed by the antisaccade task. Specifically, these prefrontal areas have been argued to be involved not only in inhibition, but also in functions such as the representation of task set when two or more tasks compete with one another for execution (Garavan, Hester, Murphy, Fassbender, \& Kelly, 2006; Herath, Klingburg, Young, Amunts, \& Roland, 2001). Although the present study did not use fMRI methods, the involvement of these areas in antisaccade performance allows a reliable interpretation of saccadic eye movements as an index of attentional control. The basic assumptions are in line with the predictions of attentional control theory (Eysenck et al., 2007).

The aim of the present study was to test the theoretical prediction that anxiety affects efficient task-switching performance, which involves reconfiguration of task sets (parameters associated with each task) in working memory to allow execution of the task appropriate to the current goal (e.g., Rubinstein, Meyer, \& Evans, 2001; for a review, see Monsell, 2003). The view that anxiety affects task-switching performance is based on two main assumptions. First, according to Eysenck et al. (2007), anxiety affects performance on tasks that involve rapid shifting between successive task sets (maintained and updated in working memory) due to the adverse effect of anxiety on top-down attentional control (Eysenck, Payne, \& Derakshan, 2005; Harris \& Cumming, 2003).

The second assumption relates to the important role of working memory capacity in successful task set reconfiguration. More specifically, the ability to successfully select and execute the goal-relevant task set (while inhibiting or terminating the irrelevant) requires efficient use of working memory resources to exercise top-down attentional control (Baddeley, Chincotta, \& Adlam, 2001; Hester \& Garavan, 2005; Kane, Bleckley, Conway, \& Engle, 2001; Meiran, 1996). Garavan et al. (2006), using fMRI, found that the DLPFC had a supervisory role in working memory functions, such as representation of task set, stimulus appraisal, and response selection. Behavioral data (Hester \& Garavan, 2005, Experiment 1) showed that increasing the number of items to be held in working memory decreased the speed at which participants switched attention from a primary working memory task to a secondary decision-making task. This suggests that diminished working memory capacity decreases the efficiency with which attentional control can be exerted.

To test this prediction systematically, we adopted the mixed antisaccade task to examine the effect of anxiety on task-switching performance. This paradigm requires participants to randomly switch between anti- and prosaccade tasks in the mixed-task block, whereas in the singletask block, they performed either the antisaccade or the prosaccade task separately. Switching performance is assessed by comparing performance on trials in which participants perform one task repeatedly (in the single-task block, or repeat trials in the mixed-task block) with trials in which they are required to randomly alternate between two tasks (mixed-task block at a general/global level or switch trials in this block at a specific/local level; see, e.g., Kray, 2006).

There is a paradoxical improvement in antisaccade performance when participants are required to switch between anti- and prosaccade trials, as compared with repeat trials. For example, Cherkasova, Manoach, Intriligator, and Barton (2002) examined residual switch cost in a randomized mixed antisaccade task (comparing latencies and error rates between the switch and the repeat trials, in the mixed-task block). They observed reduced antisaccade latencies and greater error rates in the switch trials but an effect that was not attributable to a speed-accuracy tradeoff (see also Manoach et al., 2002). Hodgson, Golding, Molyva, Rosenthal, and Kennard (2004, Experiment 1), using a more predictable presentation sequence (a switch after eight consecutive trials of one saccade type), compared antisaccade performance in the single-task and mixed-task blocks. They reported a significant reduction in antisaccade latencies in the latter, which they suggested may have been due to allocation of more attentional resources to the task in this block, "leading to a benefit in performance of antisaccade task" (p. 320).

This paradoxical improvement can be interpreted within a goal-driven controlled processing approach (see Nieuwenhuis, Broerse, Nielen, \& Jong, 2004), so that "performance variability in tasks assessing executive functions may arise from failures to fully or consistently focus at- 
tention on task requirements . . . although task requirements may be understood and remembered, they are not turned into active goals or adequately maintained as such" (p. 199), also known as goal neglect (De Jong, Berendsen, \& Cools, 1999). Hence, the paradoxical switch benefit in antisaccade latency could be due to the allocation of more attentional resources to the task requirements when the task goal is externally presented and, hence, updated on every trial (in the mixed-task block), as compared with when the task goal is presented at the beginning of a block of consecutive trials (in the single-task block) and must be maintained throughout and activated internally.

Here, we examined how anxiety affected the observed improvement in switching efficiency on the antisaccade task. Saccade latency and accuracy in the mixed-task block were compared with performance in the single-task block. Saccade performance was also compared between the switch and the repeat trials within the mixed-task block. On the basis of the predictions of the attentional control theory (Eysenck et al., 2007), we expected that HA individuals, as compared with LA individuals, would not show the improved antisaccade performance, as measured by switch cost. In other words, when shifting was involved, anxiety would interfere with the efficient allocation of attentional resources to implement the current goal relevant to the task, as measured by correct saccade latency. However, we predicted that anxiety would not impair performance effectiveness, as measured here by saccadic error rate.

\section{METHOD}

\section{Participants}

Fifty-nine participants $($ mean age $=31.9, \min =18.0 ; \max =46$, $S D=7.5$ ) were recruited by means of advertisement and university participant panels. All had corrected-to-normal vision and were allowed to wear their glasses or contact lenses. Each individual was tested in a separate laboratory session lasting approximately $40 \mathrm{~min}$ and were reimbursed $(£ 5)$ for their contribution.

\section{Cue and Target Stimuli}

The stimulus serving as cue was an oval-shaped object $\left(3.3^{\circ} \times 6^{\circ}\right)$, and the target stimulus was an arrow pointing either up or down $\left(0.6^{\circ} \times 2.3^{\circ}\right)$. These stimuli were created using the Microsoft Paint graphics application.

\section{Eyetracking Specification}

Eye movements were measured unobtrusively via a remote camera mounted below the computer monitor and using the LC Technologies Eyegaze system (LC Technology, Inc., 2003) to track the participant's eye movements. This is an infrared-based eyetracking software that generates raw gaze location data at a sampling rate of $60 \mathrm{~Hz}$, allowing eye movements to be recorded every $16.67 \mathrm{msec}$. It uses the pupil-center corneal reflection method to estimate gaze points (i.e., the intersection of the optic axis with the screen). The eyetracker is calibrated using nine fixation points, with which the software indicates whether or not valid gaze points can be calculated. The Eyegaze system accommodates several sources of error, such as head range variation and pupil diameter variation. Data are collected from the eye for which the most rapid and accurate calibration is obtained.

The presentation of the stimuli was controlled by the DMDX program (Forster \& Forster, 2003), which also recorded responses from the button box (PIO-12 interface) at $1000 \mathrm{~Hz}$, ensuring millisecond timing accuracy. The stimuli were presented in 24-bit color on a
$1,024 \times 768$ LCD monitor (ViewSonic 700 b; cell response time, $35 \mathrm{msec}$ ). The eyetracking system and DMDX were automatically synchronized at the beginning of each trial.

\section{Experimental Task}

The participants were required to complete antisaccade and prosaccade tasks presented in two blocks: single task and mixed task. In the single-task block, the participants completed trials of the same task, either anti- or prosaccade, consecutively in different blocks. In the mixed-task block, anti- and prosaccade trials were presented randomly, requiring the participants to switch between the two tasks. These tasks will be detailed below.

Anti- and prosaccade tasks. As is depicted in Figures 1A and $1 \mathrm{~B}$, a trial began with the word "Ready," followed by a central fixation symbol (subtending $0.6^{\circ}$ ). The participants were instructed to fixate it until it disappeared. In the repeat condition (Figure 1A), this fixation symbol was a white cross $\left(2^{\circ} \times 2^{\circ}\right)$. In the switch condition (Figure $1 \mathrm{~B}$ ), the fixation symbol was either a white diamond $\left(1.2^{\circ} \times 2.4^{\circ}\right)$ or a white circle $\left(1.7^{\circ}\right.$ diameter $)$ and provided instructions for the type of saccade to be performed on that trial (e.g., the diamond might indicate a prosaccade trial, and the circle an antisaccade trial). After symbol offset and a 200 -msec gap, an oval object appeared $11^{\circ}$ to either the left or the right side of the screen. The participants were required to direct their gaze either "away" (antisaccade) from the oval cue to its mirror position on the screen (without looking at the cue) or "toward" it (prosaccade).

At cue offset, an arrow either replaced the cue (on prosaccade trials) or appeared on the side of the screen opposite to the cue (on antisaccade trials). The participants were required to identify the direction of the arrow (up or down) by pressing the relevant button on the buttonpress box. ${ }^{1}$ The target arrow was shown for $100 \mathrm{msec}$.

\section{Experimental Procedure}

The participants completed the experiment in two sessions separated by a short break. In half of the experimental blocks, anti- and prosaccade trials were presented randomly (mixed-task block), and in the remaining half, anti- and prosaccade trials were presented consecutively in separate blocks (single-task block). In the single-task block, the participants were instructed as follows:

In this task, you will be asked to either look TOWARDS or AWAY FROM an object which will appear either on the left or right side of the screen. Every trial will begin with the word 'Ready' followed by a cross $(+)$. Whenever this appears look at it. When asked to look toward the object, you must look AT it VERY QUICKLY when it appears. When asked to look away, you MUST NOT look at the object but look AWAY to the side of the screen opposite the oval object VERY QUICKLY.

An arrow will then appear on the screen, you should identify the direction of the arrow (pointing UP or DOWN) using the appropriate keys on the box. You should do this VERY QUICKLY. So remember that Speed and Accuracy are very important.

And this was the same for the mixed-task block, with the following modification:

. This [the cross] will be followed by a symbol that can either be a DIAMOND or a CIRCLE. Then an oval-shaped object will be flashed on either the left or right side of the screen. When the symbol is a circle you must look AWAY to the side of the screen opposite the oval object VERY QUICKLY (You MUST NOT LOOK at the oval object at all). When the symbol is a diamond then you must look AT the oval object as soon as it appears.

An arrow will then ...

The participants were told that the purpose of the study was to examine perception of objects. After completing the consent form, the participants rated their mood on five $100-\mathrm{mm}$ visual analogue scales (VASs; 0, not at all, to 100, very much) to indicate their mood states at that point in time (e.g., Bond, Shine, \& Bruce, 1995). The dimensions included happy, irritable, sad, anxious, and comfortable states. 
A

Antisaccade Task

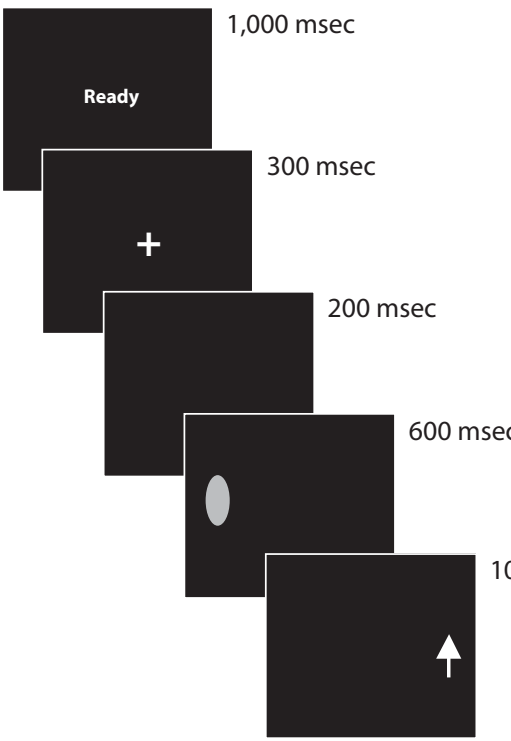

Prosaccade Task

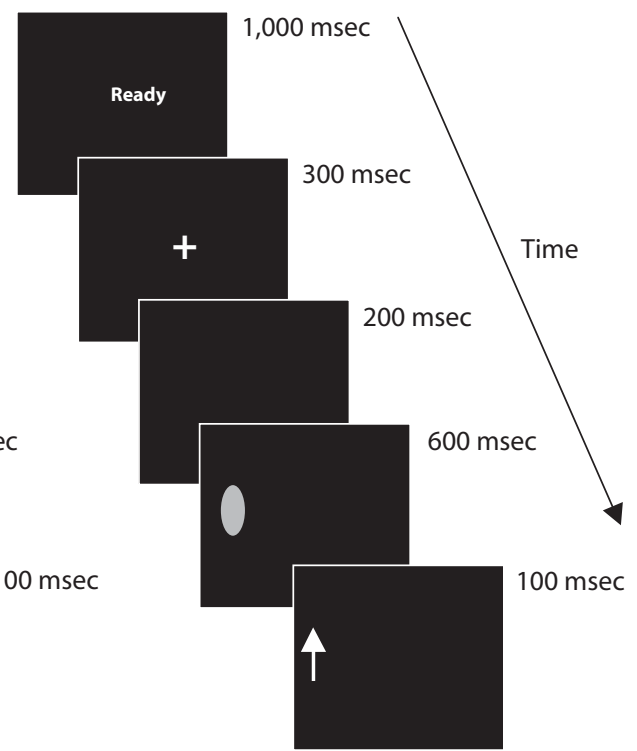

B

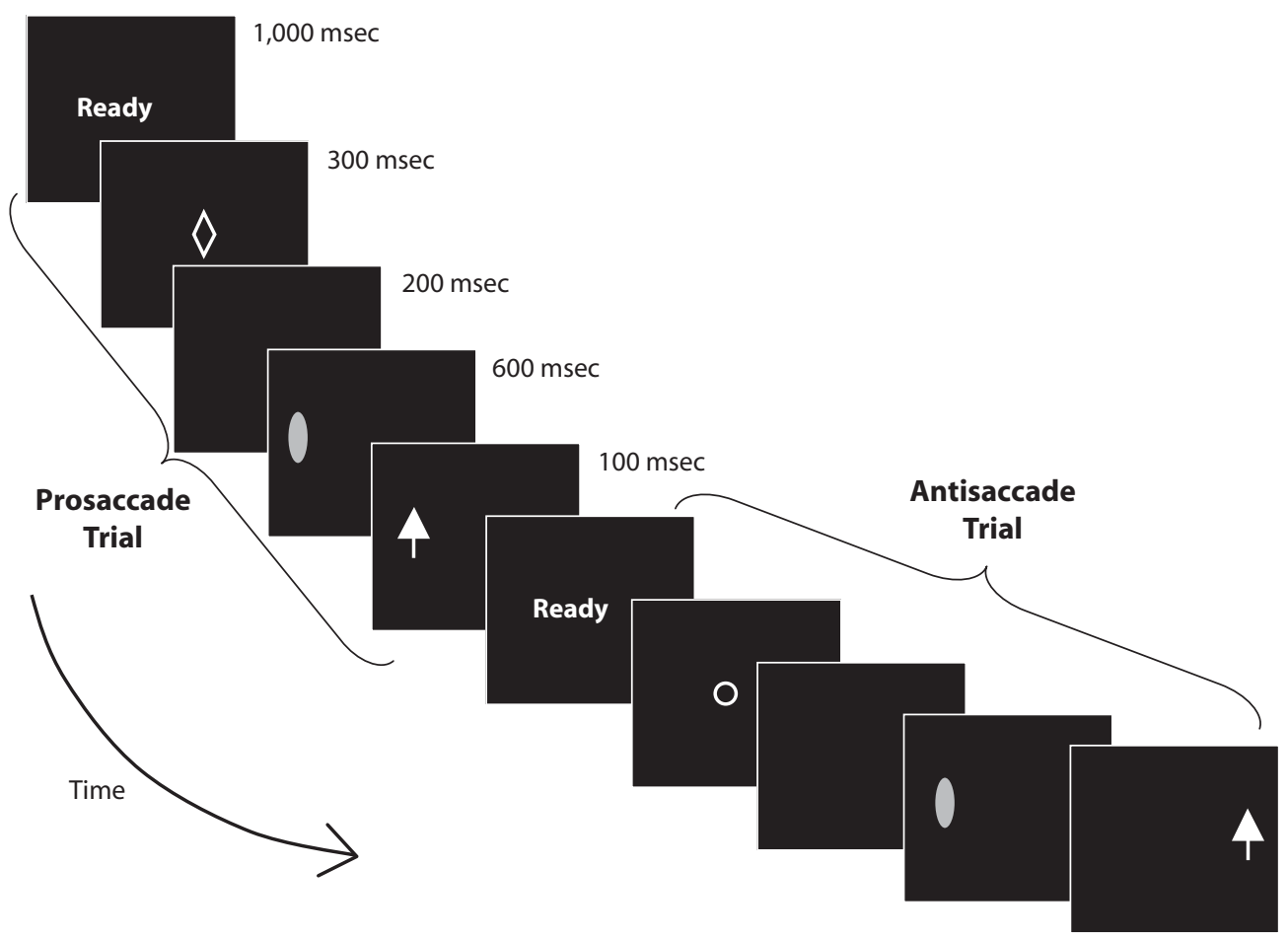

Figure 1. (A) Anti- and prosaccade tasks in the single-task block. (B) Anti- and prosaccade tasks in the mixed-task block.

The participants completed these scales at the end of the experiment also. Next, half of the participants completed the measure of trait anxiety, the State-Trait Anxiety Inventory (Spielberger, Gorsuch, Lushene, Vagg, \& Jacobs, 1983), after which they were given a distractor task involving searching for vowels in different matrices of letters. This lasted under $5 \mathrm{~min}$. The remaining participants completed the scale at the session's end.
The participants were given an overview of the eyetracking equipment, and the response buttons were indicated. They were seated directly in front of the computer monitor, with their chin placed on a chinrest (located $60 \mathrm{~cm}$ from the screen), in a dimly lit cubicle. Twenty-four practice trials were completed (12 switch, 12 repeat), at the end of which the participants were asked to verbalize the instructions in order to ensure that they had been fully understood. The 
eyetracker was calibrated. Speed and accuracy were emphasized. At the end, the participants were fully debriefed on the purpose of the study and were paid for their contribution.

The experiment comprised eight blocks of 20 experimental trials each, half of which were antisaccade and half of which were prosaccade (320 trials in total, half of which were switch and half repeat trials). The orders in which the anti- and prosaccade trials (AB-BA) and the single-task and mixed-task blocks (ABBA-BAAB) were presented were counterbalanced in a between-subjects design. The instructional meaning of the circle and diamond fixation symbols used in the mixed-task trials was also counterbalanced; hence, on half of the trials, the diamond symbol instructed an antisaccade (and the circle a prosaccade), whereas on the remaining half, it instructed a prosaccade (and the circle an antisaccade).

\section{Data Preparation}

Saccades were defined as eye movements with velocities exceeding $30^{\circ} / \mathrm{sec}$ (Massen, 2004; Reuter, Jäger, Bottlender, \& Kathmann, 2007) and amplitudes exceeding $3^{\circ}$ that were made after cue onset and before cue offset. We examined directional accuracy (percentage of error), which depended on the required response (antisaccade or prosaccade), and latency of the first correct saccade.

An incorrect saccade was defined as the first saccade after cue onset with an amplitude $>3^{\circ}$ toward the position of the cue (on antisaccade trials) or away from the cue (on prosaccade trials). The $l a-$ tency of the first correct saccade was defined as the elapsed time between the onset of the cue and the beginning of a saccade toward the correct area of interest.

We examined task-switching performance by first comparing saccade latency and error rate in the single-task block with those in the mixed-task block (in which anti- and prosaccade presentation was randomized). We calculated latency switch cost by subtracting mean correct saccade latency in the single-task block from mean correct saccade latency in the mixed-task block, a commonly used methodology. With this comparison, it is difficult to disentangle the possible contributions of cuing of task goal in the mixed-task block. In order to avoid this possible confound, we also performed trial-bytrial analysis in the mixed-task block. In this block, trials were classified as either switch (those trials preceded by a trial of the different task type) or repeat (those trials preceded by a trial of the same task type). Latency switch cost in the mixed-task block was calculated by subtracting the mean latency on repeat trials from that on switch trials. Error rates were subjected to the same procedure.

Trials were excluded from analysis if eyetracking was interrupted due to a lost pupil or if no eye movements were made by the participant. Trials on which the onset of the first saccade was shorter than $83 \mathrm{msec}$ (i.e., anticipatory) or the latency to respond was greater than $600 \mathrm{msec}$ were also excluded from analysis. These criteria resulted in the loss of $4.31 \%$ of the antisaccade and $4.38 \%$ of the prosaccade trials in the switch condition and $4.55 \%$ of the antisaccade and $4.16 \%$ of the prosaccade trials in the repeat condition. An analysis showed that data loss did not differ as a function of task or trial type.

\section{Behavioral Data}

The participants were also measured on reaction time of correct response to arrow identification. Trials with incorrect responses were removed, as were trials with reaction times less than $100 \mathrm{msec}$ or greater than $2,000 \mathrm{msec}$. Of the data, $5.2 \%$ were lost due to outliers and erroneous responses.

\section{RESULTS}

\section{Participants}

Four people had to be excluded due to poor tracking. This resulted in 55 participants in the final data set. Mean trait anxiety score as measured by the Spielberger StateTrait Anxiety Questionnaire (Spielberger et al., 1983) for the sample was $42.42(S D=8.91, \min =23, \max =64)$. We performed tercile splits on the trait anxiety questionnaire to produce low and high extreme group classifications. The LA individuals $(n=20)$ included those scoring 38 or lower, and the HA individuals $(n=21)$ included those scoring 45 or higher on this scale. Mean trait anxiety for the LA group was $33.55(S D=4.24, \min =23$, $\max =$ $38)$, and for the HA group it was $51.19(S D=5.93, \min =$ 45 , $\max =64$ ).

Analysis on the VAS scales showed that the task did not have an effect on the participants' mood. On each dimension, $2 \times 2$ mixed ANOVAs with time (before or after) as the within-subjects factor and group (LA or HA) as the between-subjects factor revealed nonsignificant main effects of time $[F \mathrm{~s}<1$ for happy, irritable, and sad; $F(1,39)=$ $1.7, p>.2$ for anxious; and $F(1,39)=1.4, p>.3$ for comfortable]. Interaction effects with group were also nonsignificant $[F$ s $<1$ for happy, irritable, anxious, and comfortable; and $F(1,39)=1.08, p>.3$ for sad].

\section{Eye Movement Data}

\section{Latency of Correct Saccades}

Single-task block versus mixed-task block. Figure 2 shows the mean correct saccade latencies for each of the anti- and prosaccade tasks in each of the single-task and mixed-task blocks. A $2 \times 2 \times 2$ mixed ANOVA with group (LA or HA individuals) as the between-subjects factor and task (anti- or prosaccade) and block type (single task or mixed task) as within-subjects factors revealed highly significant main effects of task $[F(1,39)=90.65, p<.001]$ and block type $[F(1,39)=10.34, p=.003]$, as well as highly significant block type $\times \operatorname{task}[F(1,39)=63.72, p<$ $.001]$ and block type $\times$ task $\times$ group $[F(1,39)=6.99$, $p=.01]$ interactions. The main effect of group was not significant $[F(1,39)=1.7, p=.2]$. The main effect of task showed that the participants had longer antisaccade $(M=280.59 \mathrm{msec}, S D=57.81)$ than prosaccade $(M=$

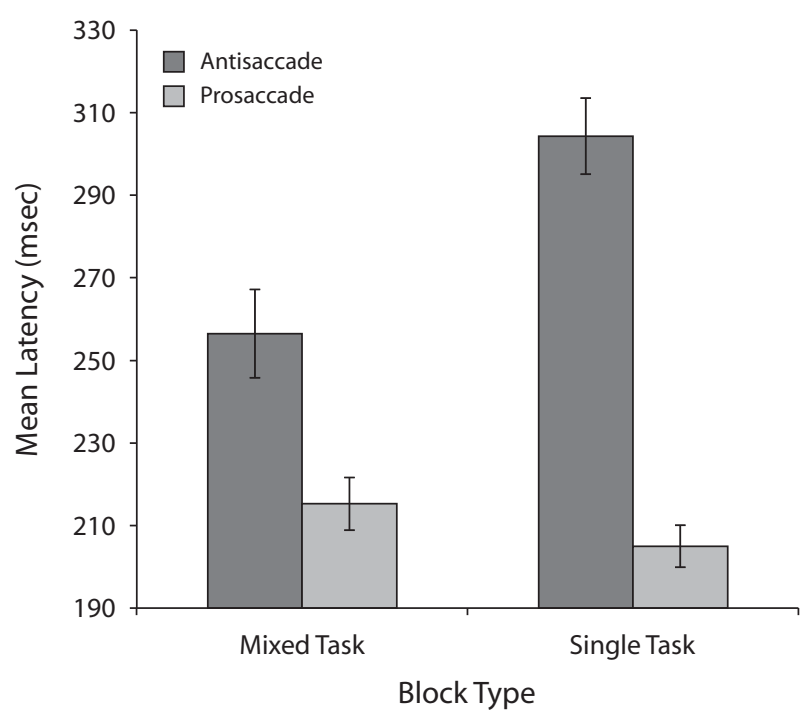

Figure 2. Mean anti- and prosaccade latencies (with standard errors) for single-task and mixed-task blocks. 
$210.32 \mathrm{msec}, S D=34.19)$ latencies. Similarly, the main effect of block type showed shorter saccade latencies in the mixed task $(M=236.21 \mathrm{msec}, S D=50.87)$ than in the single task $(M=254.87 \mathrm{msec}, S D=39.87)$. The task $\times$ block type interaction indicated that the participants had significantly shorter antisaccade latencies in the mixedtask block $(M=256.94 \mathrm{msec}, S D=70.29)$ than in the single-task block $(M=304.24 \mathrm{msec}, S D=58.3)[t(40)=$ $5.26, p<.001]$. However, in the prosaccade trials, the reverse was true $[M=215.49 \mathrm{msec}, S D=41.56$, and $M=$ $205.16 \mathrm{msec}, S D=33.03$, for mixed-task and single-task blocks, respectively; $t(40)=2.13, p=.079$ ]. These findings are consistent with those in Cherkasova et al. (2002).

Of major importance were the effects involving anxiety, as indicated by the three-way block type $\times$ task $\times$ group interaction $[F(1,39)=6.99, p=.01]$. This interaction indicated that, whereas the LA individuals exhibited shorter antisaccade latencies in the mixed-task block $(M=237.5 \mathrm{msec}, S D=66.95)$ than in the single-task block $(M=307.01 \mathrm{msec}, S D=53.6)$ [mean difference $=$ $-69.50 \mathrm{msec} ; t(19)=6.92, p<.001]$, the HA individuals showed no such improvement $(M=275.45 \mathrm{msec}, S D=$ 69.89 , for the mixed task and $M=301.61 \mathrm{msec}, S D=$ 63.66 , for the single task) $[$ mean difference $=26.16 \mathrm{msec}$; $t(20)=1.9, p>.08]$. If we define switch cost as the mean latency difference between the single-task and the mixedtask blocks, we can see in Figure 3 a switch benefit in antisaccade latency for LA individuals, but not HA individuals. When we examined prosaccade latencies, there were no differences between the mixed-task block $(M=$ $205.33 \mathrm{msec}, S D=34.76)$ and the single-task block $(M=$ $197.48 \mathrm{msec}, S D=28.45)$ in the LA group $[t(19)=1.29$, $p=.2]$, and the same pattern was observed in the HA group (for the mixed-task block, $M=225.16 \mathrm{msec}, S D=$ 45.88; for the single-task block, $M=212.47 \mathrm{msec}, S D=$ 36.03) $[t(20)=1.67, p>.1]$.

Group differences were examined on switch cost in both anti- and prosaccade performance. The groups differed on antisaccade switch cost $[t(39)=2.57, p=.01]$, but not on prosaccade switch $\operatorname{cost}(t<1)$.

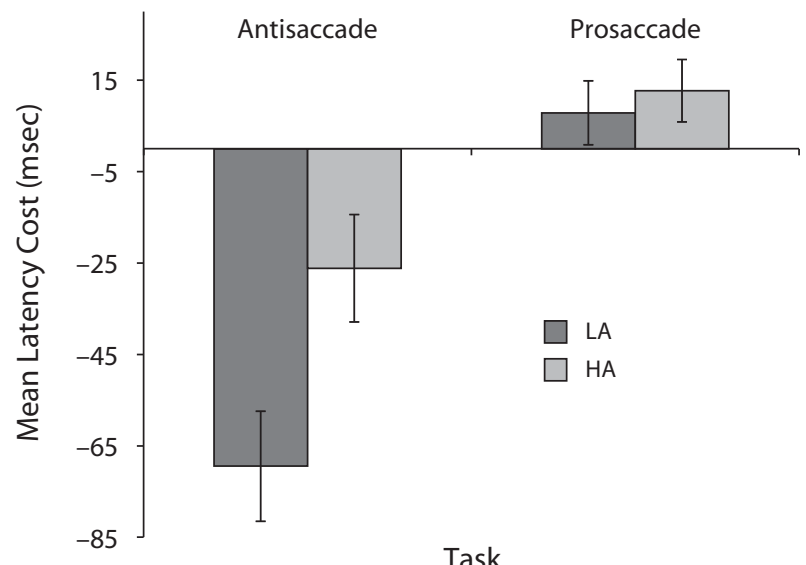

Figure 3. Latency switch costs (and standard errors) for lowanxious (LA) and high-anxious (HA) individuals on both antiand prosaccade trials: Single-task versus mixed-task blocks.

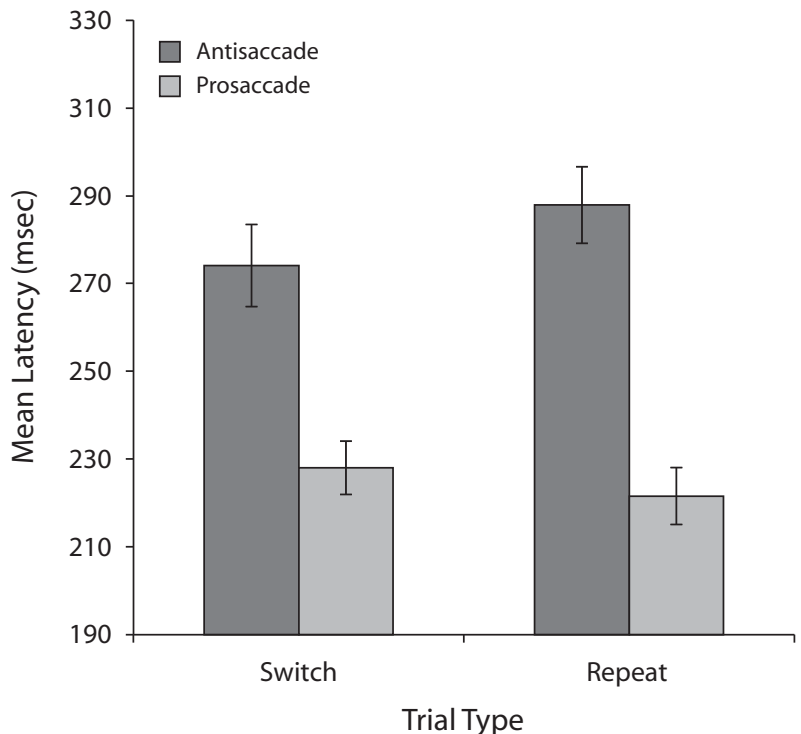

Figure 4. Mean anti- and prosaccade latencies (with standard errors) for the repeat and switch trials in the mixed-task block.

\section{Switch versus repeat trials in the mixed-task block.}

Figure 4 shows mean correct saccade latencies for each of the anti- and prosaccade tasks on the switch and repeat trials in the mixed-task block. A $2 \times 2 \times 2$ mixed model ANOVA with group as the between-subjects factor and task (anti or pro) and trial type (switch or repeat) as within-subjects factors revealed a highly significant main effect of task $[F(1,39)=57.18, p<.001]$, as well as highly significant trial type $\times$ task $[F(1,39)=7.78, p=.008]$ and trial type $\times$ task $\times$ group $[F(1,39)=6.99, p=.01]$ interactions. The main effect of task showed that, in the mixed-task block, the participants had longer antisaccade $(M=281.44 \mathrm{msec}, S D=55.26)$ than prosaccade $(M=$ $225.03 \mathrm{msec}, S D=38.43)$ latencies. The task $\times$ trial type interaction indicated that the participants had shorter antisaccade latencies when switching between anti- and prosaccade tasks $(M=274.72 \mathrm{msec}, S D=64.47)$ than when antisaccade trials were repeated $(M=288.16 \mathrm{msec}, S D=$ 56.31) $[t(40)=2.19, p=.03]$. On prosaccade trials, the participants became somewhat slower when required to switch $(M=228.18 \mathrm{msec}, S D=39.01)$ than when prosaccade trials were repeated ( $M=221.87 \mathrm{msec}, S D=42.63)$, but this effect was not significant $[t(40)=1.45, p=.15]$.

More important to the purposes of the present study is the effect of anxiety on performance, as suggested by the significant trial type $\times$ task $\times$ group interaction. This interaction showed that whereas LA individuals became significantly faster on antisaccade trials when switching between antiand prosaccade trials $(M=248.64 \mathrm{msec}, S D=64.2)$ than when antisaccade trials were repeated $(M=276.7 \mathrm{msec}$, $S D=53.60$ ) [mean difference of $-28.05 \mathrm{msec} ; t(19)=$ $3.02, p=.007]$, the HA individuals were not affected on antisaccade performance $[M=299.56 \mathrm{msec}, S D=55.39$, when switching between trials, and $M=299.07 \mathrm{msec}$, $S D=57.92$, when antisaccade trials were repeated; mean difference $=0.49 ; t(20)<1]$. If we define mixed-task 


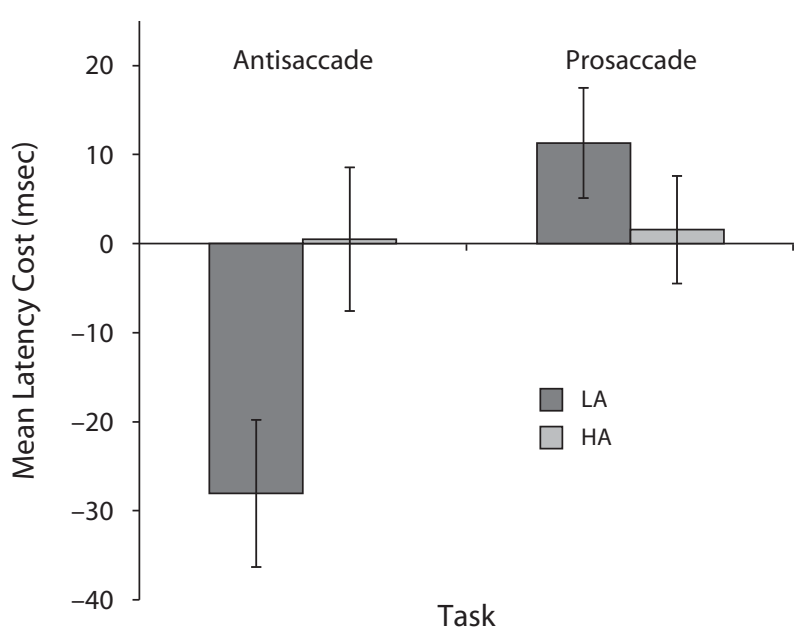

Figure 5. Latency switch costs (and standard errors) for lowanxious (LA) and high-anxious (HA) individuals on both antiand prosaccade trials: Repeat versus switch trials in the mixedtask block.

switch cost as the mean latency difference between repeat and switch trials within the mixed-task block, Figure 5 shows that LA individuals exhibited a switch benefit in antisaccade latency, whereas HA individuals did not. When prosaccade performance is examined, the LA individuals became somewhat slower on these trials when required to switch between anti- and prosaccades $(M=221.29 \mathrm{msec}$, $S D=33.31)$ than when prosaccades were repeated $(M=$ $209.99 \mathrm{msec}, S D=32.52)[t(19)=2.37, p=.03]$. However, the HA individuals were not affected on prosaccade performance $[M=234.74 \mathrm{msec}, S D=43.56$, when switching between trials; and $M=233.19 \mathrm{msec}, S D=48.52$, when prosaccade trials were repeated; $t(20)<1]$.

Group differences were examined on switch costs for both anti- and prosaccade performance. The groups differed on antisaccade switch cost $[t(39)=2.47, p=.01]$, but not on prosaccade switch cost $[t(39)=1.12, p=.28]$.

It was predicted that anxiety would have a negative impact on switching because efficient performance on the switching tasks depends on the availability of working memory resources, and since anxiety diminishes available resources, performance on such cognitive tasks would be expected to deteriorate. The presented findings collectively support this view.

\section{Percentage of Saccade Error}

Single-task block versus mixed-task block. Figure 6 depicts the error rates for each of the LA and HA groups on the anti- and prosaccade tasks for the single-task and mixed-task blocks. The percentage of trials excluded from the analysis did not differ between the two groups $[t(39)<1]$. The participants made more saccadic errors on the antisaccade task $(M=15.071 \%, S D=15.49)$ than on the prosaccade task $(M=3.5 \%, S D=5.3)$. Similarly, the participants were generally worse in the mixed-task block $(M=12.48 \%, S D=12.7)$ than in the singletask block $(M=6.16 \%, S D=6.43)$. This observation was confirmed by a $2 \times 2 \times 2$ mixed ANOVA with task and block type as within-subjects factors and group as a between-subjects factor, which yielded significant main effects of task $[F(1,39)=28.83, p<.001]$ and block type $[F(1,39)=24.53, p<.001]$. The two-way task $\times$ block type interaction and the three-way task $\times$ block type $\times$ group interaction were not significant.

Switch versus repeat trials in the mixed-task block. Figure 7 depicts the error rates for each of the LA and HA groups on the anti- and prosaccade tasks for the repeat and switch trials. The percentage of trials excluded from the analysis did not differ between the two groups $[t(39)<1]$. The participants had higher error rates on the antisaccade task $(M=26.99 \%, S D=12.83)$ than on the prosaccade task $(M=11.28 \%, S D=8.51)$. In a similar way, the participants had higher error rates when required to switch $(M=20.70 \%, S D=10.64)$ than when trials were repeated $(M=17.57 \%, S D=10.30)$. This observation was supported by a $2 \times 2 \times 2$ mixed ANOVA on percentage of saccade error, with group as a between-subjects factor and task and trial type as within-subjects factors, which revealed a significant main effect of task $[F(1,39)=126.07$, $p<.001]$ and trial type $[F(1,39)=10.98, p<.003]$. The two-way task $\times$ trial type interaction was not significant, nor was the three-way task $\times$ trial type $\times$ group interaction (both $F_{\mathrm{s}}<1$ ).

\section{Relation Between Saccade Latency and Error Rate Switch Cost}

Despite the nonsignificant task $x$ trial type and task $X$ trial type $\times$ group interactions on analysis of error rates, we decided to conduct a further analysis to examine whether the shorter antisaccade latencies, as observed on the switch trials, and the higher error rates on these trials could be explained in terms of a speed-accuracy trade-off. We examined this relationship for each group separately, by analyzing performance on the single task with that on the mixed task and by analyzing this relationship in the mixed block only. The relationship was not significant for either the LA or the HA group $(r=-.11, p=.6$, and $r=$

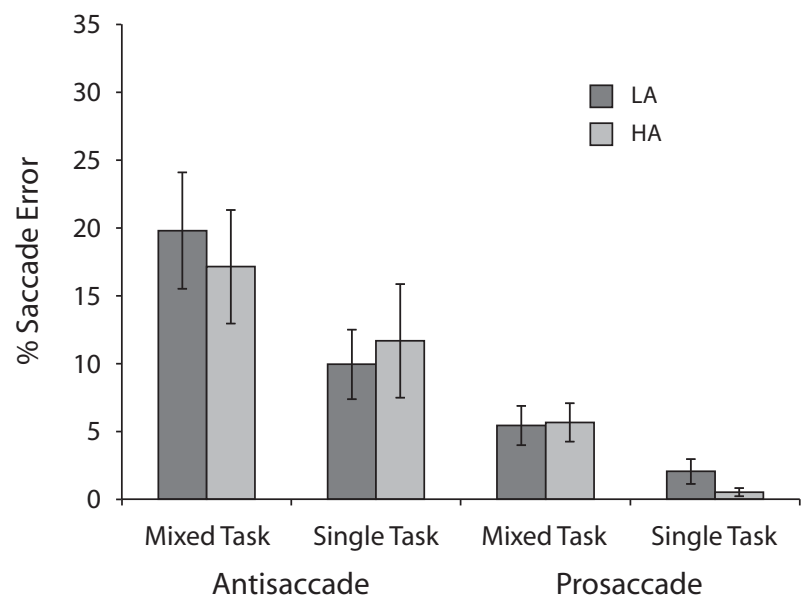

Figure 6. Percentages of anti- and prosaccade errors (with standard errors) in the single-task and mixed-task blocks. LA, low anxious; HA, high anxious. 


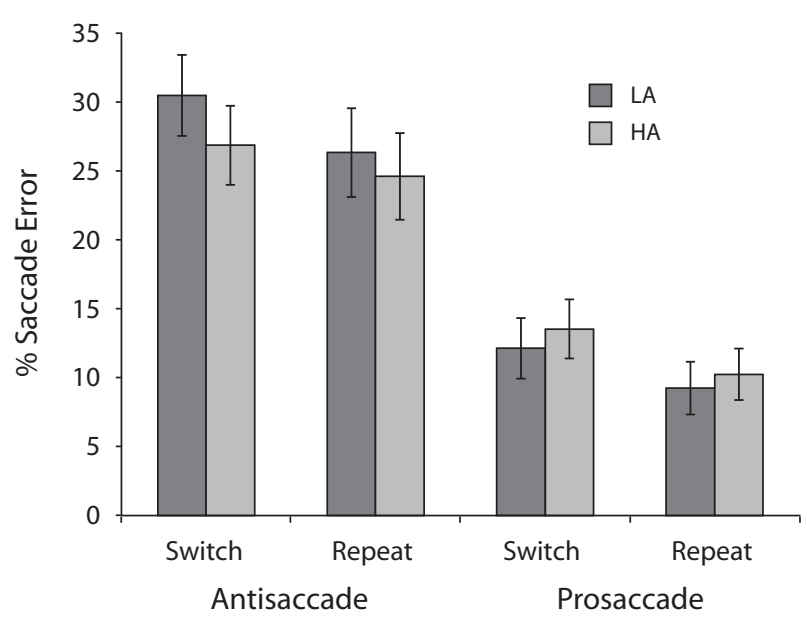

Figure 7. Percentages of anti- and prosaccade errors (with standard errors) in switch and repeat trials in the mixed-task block. LA, low anxious; HA, high anxious.

$-.02, p=.9$, respectively) when we compared performance on the single task with that on the mixed task. Similar effects were found when we examined the relationship in the mixed block only $(r=-.34, p=.14$, and $r=-.12$, $p=.6$, for the LA and the HA groups, respectively). These results indicate that saccade latency improvement was not simply the result of a speed-accuracy trade-off.

\section{Behavioral Data}

The participants were generally slower to identify the direction of the target arrow in the mixed-task block $(M=$ $487.92 \mathrm{msec}, S D=121.84$ ) than in the single-task block $(M=463.57 \mathrm{msec}, S D=93.5)$ and were slower on the antisaccade task $(M=482.44 \mathrm{msec}, S D=108.55)$ than on the prosaccade task $(M=469.05 \mathrm{msec}, S D=107.05)$. A $2 \times 2 \times 2$ mixed ANOVA on mean reaction time to target arrow, with task and block type as within-subjects factors and group as a between-subjects factor, revealed a significant main effect of block type $[F(1,39)=11.42, p<.002]$ and task $[F(1,39)=5.31, p=.03]$. No other effects or interactions were found with group and task $\left(F_{\mathrm{s}}<1\right)$.

\section{DISCUSSION}

Our results support the predicted effects, with greater error rates and longer latencies on the antisaccade than on the prosaccade task. The participants exhibited the predicted switch benefit in antisaccade latency when required to switch between anti- and prosaccade trials (mixed-task block), as compared with when they completed anti- and prosaccade tasks separately in blocks of repeated trials (single-task blocks). The paradoxical improvement in antisaccade latency is consistent with the results of previous research (e.g., Barton et al., 2002; Cherkasova et al., 2002; Hodgson et al., 2004; Manoach et al., 2002), in which similar paradoxical reductions in antisaccade latencies were reported when participants were required to switch.

The improvement in antisaccade latency observed in the present study can be associated with the precuing of the task goal on every trial in the mixed block, as compared with the single-task block (see Kray, 2006; Nieuwenhuis et al., 2004). In the mixed-task block, precuing facilitated task set reconfiguration and allowed attentional resources to be allocated to the current task, resulting in shorter antisaccade latencies. In the single-task block, however, the participants had to actively maintain and trigger the task goal in working memory throughout each anti- and prosaccade block, in turn resulting in slower task set reconfiguration and, hence, slower generation of a correct antisaccade.

Of major importance to the aims of the present experiment were the findings regarding the effect of anxiety in relation to switching benefit on correct antisaccade latencies. Comparing antisaccade performance in the singletask and the mixed-task blocks showed that LA individuals exhibited the expected switch benefit in correct antisaccade latencies but that HA individuals showed no significant improvement. However, the two groups did not differ in terms of their performance on prosaccade trials. The important group difference points to a diminished ability in HA individuals to utilize the cue to facilitate task set reconfiguration. Within the framework of the attentional control theory, these findings suggest that these individuals are less able to exercise top-down attentional control to efficiently shift attentional resources according to new task demands. This failure to use top-down control efficiently can be attributed to diminished working memory capacity in HA individuals. Indeed, previous research emphasizes the critical role of working memory capacity in task set reconfiguration, which determines successful task-switching performance (e.g., Baddeley et al., 2001; Hester \& Garavan, 2005; Kane et al., 2001; Logan, 2004).

However, in order to ensure the validity of the conclusions based on these findings, it was crucial to disentangle the possible contribution of precuing (in the mixed-task block) from that of task switching in anti- and prosaccade performance. In other words, it is unclear whether the observed performance improvement is a true reflection of the effect of anxiety on task switching in this paradigm or simply the result of a confound associated with cuing task goal in the mixed-task, but not in the single-task, block.

To do this, trial-by-trial analyses were conducted comparing performance on repeat and switch trials in the mixed-task block. The results replicated the paradoxical improvement in antisaccade latencies observed when performance in the single-task block was compared with that in the mixed-task block. More specifically, the participants exhibited a switch benefit in antisaccade latencies within the mixed-task block when the antisaccade trial was preceded by a prosaccade trial (switch trial), as compared with when it was preceded by a trial of the same task type (repeat trial). Crucially, this improvement was found to be associated solely with the antisaccade performance of LA individuals, whereas HA individuals exhibited no such improvement. These findings have important implications for understanding the underlying mechanisms by which anxiety affects task-switching performance.

According to the attentional control theory (Eysenck et al., 2007), successful switching performance requires that attentional control be exercised in a positive way to 
allocate attentional resources to the task relevant to the current goal. In the mixed-task block, switching from a prosaccade task to an antisaccade task facilitated antisaccade performance in the LA individuals, but not in the HA individuals, despite the explicit presentation of the task goal on every trial. When prosaccade performance is examined, on the other hand, LA individuals are much slower when required to switch to a prosaccade task than when prosaccades are repeated. The HA group was again unaffected by switching on these trials. The key to understanding these group differences may lie in an understanding of the effect of performing an attentionally demanding cognitive task (such as task switching) in conjunction with antisaccade performance. A recent study in which precisely this relationship was looked at (Kristjansson, Chen, \& Nakayama, 2001) showed that antisaccade performance improved significantly when it was performed simultaneously with a demanding perceptual task. The authors reasoned that the simultaneous performance of other attention-requiring tasks interferes with the programming of a reflexive response, both facilitating antisaccade performance and delaying prosaccade performance. They concluded that in the antisaccade task, "less attention is more."

In light of these findings and the findings of the present study, it is therefore conceivable that task switching facilitates antisaccade performance but diminishes prosaccade performance. Providing support for the assumptions of the attentional control theory, the present findings suggest that HA individuals are unable to exercise efficient top-down attentional control to distribute attentional resources according to task demands. This is in line with neurobiological evidence showing decreased activation in the DLFPC and the VLFPC, believed to be involved in modulation of top-down attentional control in HA individuals. Bishop (2007) reported that high levels of anxiety were associated with reduced recruitment of these areas and argued that this is indicative of a diminished ability in HA individuals to recruit these areas to augment attentional control.

Another important observation in the present study was the lack of anxiety-related effects on saccade accuracy (on both single-task vs. mixed-task and repeat vs. switch trials within the mixed-task block), despite significant effects on correct antisaccade latency. This finding is fully consistent with the prediction of the attentional control theory that anxiety is more likely to impair performance efficiency than performance effectiveness. The lack of anxiety-related differential effects on antisaccade accuracy is consistent with previous findings (Derakshan et al., in press; Derakshan \& Eysenck, 1998; Elliman, Green, Rogers, \& Finch, 1997). For example, Elliman et al. found no effect of anxiety on the number of correct responses on a sustained attention task. However, HA individuals took longer to respond, which was attributed to greater effort being exerted in performing the task and, hence, decreased performance efficiency.

As a final point, in this article, we have assumed that correct antisaccade performance reflects the individual participant's level of attentional control (e.g., Ettinger et al., 2008). However, an alternative assumption is that the control of eye movements in the antisaccade task re- flects some form of behavioral inhibition that may or may not be linked with attention. A third possibility is that there is an interaction between attentional control and behavioral inhibition in which attentional control facilitates behavioral inhibition. Given the wealth of data on the neural correlates of antisaccade performance (see Ettinger et al., 2008), we argue that saccadic eye movements in the antisaccade task are reliable indices of attentional control. We emphasize the importance of attentional control in understanding antisaccade performance and, at the same time, accept the need for more research in identifying the role of behavioral inhibition.

Overall, the findings of the present experiment show that anxiety diminishes the commonly exhibited switching benefit in correct antisaccade latency, suggesting that when shifting, HA individuals cannot implement goaldirected top-down control to allocate attentional resources to the current task demands. Furthermore, anxiety did not have an effect on directional accuracy. These observations are precisely in line with the predictions of the attentional control theory (Eysenck et al., 2007), providing a more direct assessment of the underlying mechanisms with which anxiety impairs attentional control when the shifting function is required.

The present findings indicate that anxiety impairs efficient shifting of attentional resources to task demands in the absence of threat. Theoretically, it is important to examine how threat affects task-switching performance in HA and LA individuals. There is much research in support of the association between anxiety and increased attentional bias toward threatening stimuli (e.g., Fox, Russo, Bowles, \& Dutton, 2001; Fox, Russo, \& Dutton, 2002; see Bar-Haim et al., 2007 , for a review). The attentional control theory predicts that the adverse effect of anxiety on task performance will be greater in the presence of task-irrelevant threat-related material. We are currently investigating attentional control in the presence of emotional stimuli. Finally, it is important to investigate attentional control as measured through the antisaccade task in other neuropsychiatric conditions, such as psychosis and depression. Given the high correlation between anxiety and depression, it will be useful for future research to attempt to disentangle the unique contributions of these conditions in terms of attentional control. Our lab is currently investigating the mechanisms underlying attentional control with respect to depression.

\section{AUTHOR NOTE}

The present work was supported by an ESRC Ph.D. studentship awarded to T.L.A. and was carried out under the supervision of N.D. Correspondence concerning this article should be addressed to N. Derakshan, Attention and Emotion Lab, School of Psychology, Birkbeck College, University of London, Malet Street, London WC1E 7HX, England (e-mail: n.derakhshan@bbk.ac.uk).

\section{REFERENCES}

Baddeley, A. [D.] (1986). Working memory. Oxford: Oxford University Press, Clarendon Press.

Baddeley, A. D., Chincotta, D., \& Adlam, A. (2001). Working memory and the control of action: Evidence from task switching. Journal of Experimental Psychology: General, 130, 641-657.

Bar-Haim, Y., Lamy, D., Pergamin, L., BaKermans-Kranenburg, M. J., 
\& VAN IJZENDOORN, M. H. (2007). Threat-related attentional bias in anxious and nonanxious individuals: A meta-analytic study. Psychological Bulletin, 133, 1-24.

Barton, J. J. S., Cherkasova, M. V., Lindgren, K. A., Goff, D. C., Intriligator, J., \& ManoACh, D. S. (2002). Antisaccade and task switching: Studies of control processing in saccadic function in normal subjects and schizophrenic patients. In H. J. Kaminsk \& R. J. Leigh (Eds.), Neurobiology of eye movements: From molecules to behavior (Annals of the New York Academy of Sciences, Vol. 956, pp. 250-263). New York: New York Academy of Sciences.

BisHOP, S. J. (2007). Neurocognitive mechanisms of anxiety: An integrative account. Trends in Cognitive Sciences, 11, 307-316.

Bishop, S. [J.], Duncan, J., Brett, M., \& Lawrence, A. D. (2004). Prefrontal cortical function and anxiety: Controlling attention to threat-related stimuli. Nature Neuroscience, 7, 184-188.

Bond, A. J., Shine, P., \& Bruce, M. (1995). Validation of visual analogue scales in anxiety. International Journal of Methods in Psychiatric Research, 5, 1-10.

Cherkasova, M. V., Manoach, D. S., Intriligator, J. M., \& BarTON, J. J. S. (2002). Antisaccades and task-switching: Interactions in controlled processing. Experimental Brain Research, 144, 528-537.

Corbetta, M., \& Shulman, G. L. (2002). Control of goal-directed and stimulus-driven activation in the brain. Nature Reviews Neuroscience, 3, 201-215.

De Jong, R., Berendsen, E., \& Cools, R. (1999). Goal neglect and inhibitory limitations: Dissociable causes of interference effects in conflict situation. Acta Psychologica, 101, 379-394.

Derakshan, N., Ansari, T. L., Hansard, M., Shoker, L., \& EySENCK, M. W. (in press). Anxiety and inhibition: An investigation using the antisaccade task. Experimental Psychology.

DERAKSHAN, N., \& EYSENCK, M. W. (1998). Working memory capacity in high trait-anxious and repressor groups. Cognition \& Emotion, 12, 697-713.

Derryberry, D., \& Reed, M. A. (2002). Anxiety-related attentional biases and their regulation by attentional control. Journal of Abnormal Psychology, 111, 225-236.

Elliman, N. A., Green, M. W., Rogers, P. J., \& Finch, G. M. (1997). Processing-efficiency theory and the working-memory system: Impairments associated with sub-clinical anxiety. Personality \& Individual Differences, 23, 31-35.

Ettinger, U., Ffytche, D. H., Kumari, V., Kathmann, N., ReuTER, B., Zelaya, F., \& Williams, S. C. R. (2008). Decomposing the neural correlates of antisaccade eye-movements using event-related fMRI. Cerebral Cortex, 18, 1148-1159.

EysenCK, M. W., \& CALVo, M. G. (1992). Anxiety and performance: The processing efficiency theory. Cognition \& Emotion, 6, 409-434.

Eysenck, M. W., Derakshan, N., Santos, R., \& Calvo, M. G. (2007). Anxiety and cognitive performance: The processing efficiency theory. Emotion, 7, 336-353.

Eysenck, M. W., Payne, S., \& Derakshan, N. (2005). Trait anxiety, visuospatial processing, and working memory. Cognition \& Emotion, 19, 1214-1228.

Forster, K. I., \& Forster, J. C. (2003). DMDX: A Window display program with millisecond accuracy. Behavior Research Methods, Instruments, \& Computers, 35, 116-124.

Fox, E., Russo, R., Bowles, R., \& Dutton, K. (2001). Do threatening stimuli draw or hold visual attention in subclinical anxiety? Journal of Experimental Psychology: General, 130, 681-700.

Fox, E., Russo, R., \& Dutton, K. (2002). Attentional bias for threat: Evidence for delayed disengagement from emotional faces. Cognition \& Emotion, 16, 355-379.

Garavan, H., Hester, R., Murphy, K., Fassbender, C., \& Kelly, C. (2006). Individual differences in the functional neuroanatomy of inhibitory control. Brain Research, 1105, 130-142.

Hallet, P. E. (1978). Primary and secondary saccades to goals defined by instructions. Vision Research, 18, 1279-1296.

HARRIS, L. M., \& CUMming, S. R. (2003). An examination of the relationship between anxiety and performance on prospective and retrospective memory tasks. Australian Journal of Psychology, 55, 51-55.
Herath, P., Klingburg, T., Young, J., Amunts, K., \& Roland, R. (2001). Neural correlates of dual task interference can be dissociated from those of divided attention: An fMRI study. Cerebral Cortex, 11, 796-805.

Hester, R., \& Garavan, H. (2005). Working memory and executive function: The influence of content and load on the control of attention. Memory \& Cognition, 33, 221-233.

Hodgson, T. L., Golding, C., Molyva, D., Rosenthal, C. R., \& KenNARD, C. (2004). Eye movement during task switching: Reflexive, symbolic, and affective contributions to response selection. Cognitive Neuroscience, 16, 318-330.

Hutton, S. B., \& Ettinger, U. (2006). The antisaccade task as a research tool in psychopathology: A critical review. Psychophysiology, 43, 302-313.

Kane, M. J., Bleckley, M. K., Conway, A. R. A., \& Engle, R. W. (2001). A controlled-attention view of working-memory capacity. Journal of Experimental Psychology: General, 130, 169-183.

KRAY, J. (2006). Task-set switching under cue-based versus memorybased switching conditions in younger and older adults. Brain Research, 1105, 83-92.

Kristjansson, A. J., Chen, Y., \& Nakayama, K. (2001). Less attention is more, in preparation of antisaccade but not prosaccades. Nature Neuroscience, 4, 1037-1042.

LC Technology, Inc. (2003). Eyegaze development system: Development manual. Fairfax, VA: Author.

LoGAN, G. D. (2004). Working memory, task switching, and executive control in task span procedures. Journal of Experimental Psychology: General, 133, 218-236.

Manoach, D. S., Lindgren, K. A., Cherkasova, M. V., Goff, D. C., Halpern, E. F., Intriligator, J., \& Barton, J. J. S. (2002). Schizophrenic patients show deficit inhibition but intact task-switching on saccadic tasks. Biological Psychiatry, 51, 816-826.

Massen, C. (2004). Parallel programming of exogenous and endogenous components in the antisaccade task. Quarterly Journal of Experimental Psychology, 57A, 475-498.

Meiran, N. (1996). Reconfiguration of processing mode prior to task performance. Journal of Experimental Psychology: Learning, Memory, \& Cognition, 22, 1423-1442.

Miyake, A., Friedman, N. P., Emerson, M. J., Witzki, A. H., HowERTER, A., \& WAGER, T. D. (2000). The unity and diversity of executive functions and their contributions to complex "frontal lobe" tasks: A latent variable analysis. Cognitive Psychology, 41, 49-100.

Monsell, S. (2003). Task switching. Trends in Cognitive Sciences, 7, 134-140.

Nieuwenhuis, S., Broerse, A., Nielen, M. M. A., \& Jong, R. D. (2004). A goal activation approach to the study of executive function: An application to antisaccade tasks. Brain \& Cognition, 56, 198-214.

Pashler, H., Johnston, J. C., \& Ruthruff, E. (2001). Attention and performance. Annual Review of Psychology, 52, 629-651.

Reuter, B., JäGer, M., Bottlender, R., \& KathmanN, N. (2007). Impaired action control in schizophrenia: The role of volitional saccade initiation. Neuropsychologia, 45, 1840-1848.

Rubinstein, J. S., Meyer, D. E., \& Evans, J. E. (2001). Executive control of cognitive processes in task switching. Journal of Experimental Psychology: Human Perception \& Performance, 27, 763-797.

Spielberger, C. C., Gorsuch, R. L., Lushene, R., VAGG, P. R., \& JACoBs, G. A. (1983). Manual for the State-Trait Anxiety Inventory. Palo Alto, CA: Consulting Psychologists Press.

Wickens, C. D. (1984). Processing resources in attention. In R. Parasuraman \& R. Davies (Eds.), Varieties of attention (pp. 63-101). New York: Academic Press.

\section{NOTE}

1. The main purpose that the arrow identification task served was to ensure the participants' engagement in the main task.

(Manuscript received November 30, 2007; revision accepted for publication March 30, 2008.) 